\title{
A Curious Case of Pericardiohydroptysis
}

\author{
Khalid Mohammad ${ }^{\mathrm{a}, \mathrm{c}}$, Yousef Shweihat ${ }^{\mathrm{a}}$, \\ Gareth $\mathrm{H}$ Tobler ${ }^{\mathrm{b}}$, Thaddeus Bartter ${ }^{\mathrm{a}}$
}

\begin{abstract}
Rupture of the heart or pericardium is a rare clinical finding almost always associated with trauma to the chest. Non-traumatic pericardial rupture into the lung parenchyma and pleural space has not been reported in literature. Hence the progression or natural course is not known and the optimal management not defined. We report a case of a 65-year-old male patient with past medical history of hypertension and alcohol abuse who presented with complaints of fever, and sharp retrosternal chest pain and was found to have rupture of the pericardium into the right pleural space and lung parenchyma. The diagnosis was established with a computed tomography scan of the chest and a cardiac magnetic resonance imaging. Routine investigation and a surgical biopsy of the pericardium could not establish a cause for the pericardial rupture. He was treated as idiopathic pericarditis with improvement over time, and a repeat echocardiogram showed resolution of the pericardial effusion. More than a year after the initial presentation, the patient is stable with no complications. From the sequence of events, we presume that the productive cough which immediately preceded this diagnosis represented the coughing up of pericardial fluid (pericardiohydroptysis).
\end{abstract}

Keywords: Pericarditis; Pericardial rupture; Pericardiohydroptysis

\section{Introduction}

Rupture of the heart or pericardium is a rare clinical finding

Manuscript accepted for publication February 21, 2013

${ }^{a}$ Department of Pulmonary and Critical Care Medicine, University of Arkansas for Medical Sciences, Little Rock, AR, USA

${ }^{\mathrm{b}}$ Department of Cardiovascular and Thoracic Surgery, University of Arkansas for Medical Sciences, Little Rock, AR, USA

${ }^{\mathrm{c} C}$ Corresponding author: Khalid Mohammad, Department of Pulmonary and Critical Care Medicine, University of Arkansas for Medical

Sciences, Little Rock, AR, USA. Email: khalidmdmdoc@gmail.com

doi: http://dx.doi.org/10.4021/jmc1138w almost always associated with trauma to the chest. It is most often associated with other major injuries and is usually diagnosed postmortem [1]. We present a case of non-traumatic pericardial rupture into the right pleural space and lung parenchyma.

\section{Case Report}

A 65-year-old male with past medical history of hypertension and alcohol abuse presented to the hospital with complaints of one month of fever with intermittent chills, shortness of breath, and sharp retrosternal chest pain. He had been treated at an outside hospital first with antibiotics and then with two weeks of intravenous and oral steroids for a diagnosis of pericarditis. On arrival, he stated that over the preceding twenty-four hours he had coughed up reddish to dark-brown sputum which had initially been copious but had subsided by the time of our examination. On physical examination, the patient appeared well-nourished. He was febrile $\left(38.3^{\circ} \mathrm{C}\right)$ with pulse $82 / \mathrm{min}$, blood pressure $148 / 75 \mathrm{mmHg}$, respiratory rate 20 , and an oxygen saturation of $94 \%$ on supplemental oxygen. On chest auscultation, air entry was decreased at both bases. Cardiac examination was unremarkable.

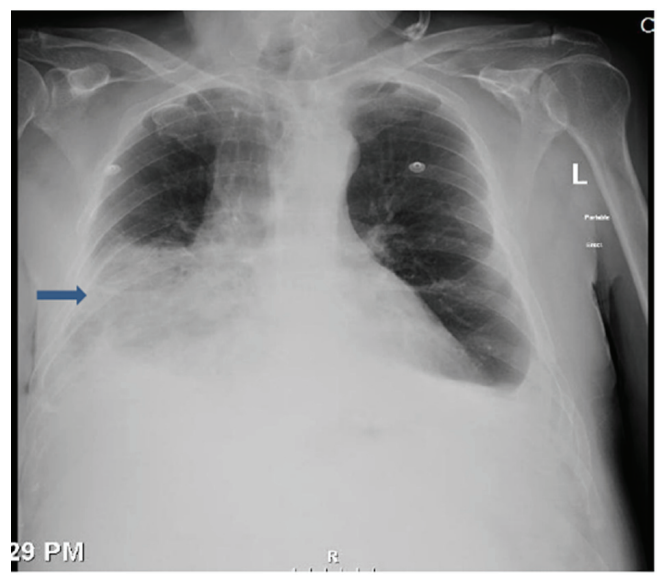

Figure 1. Bilateral pleural effusion with airspace disease involving the right middle and lower lobes. 

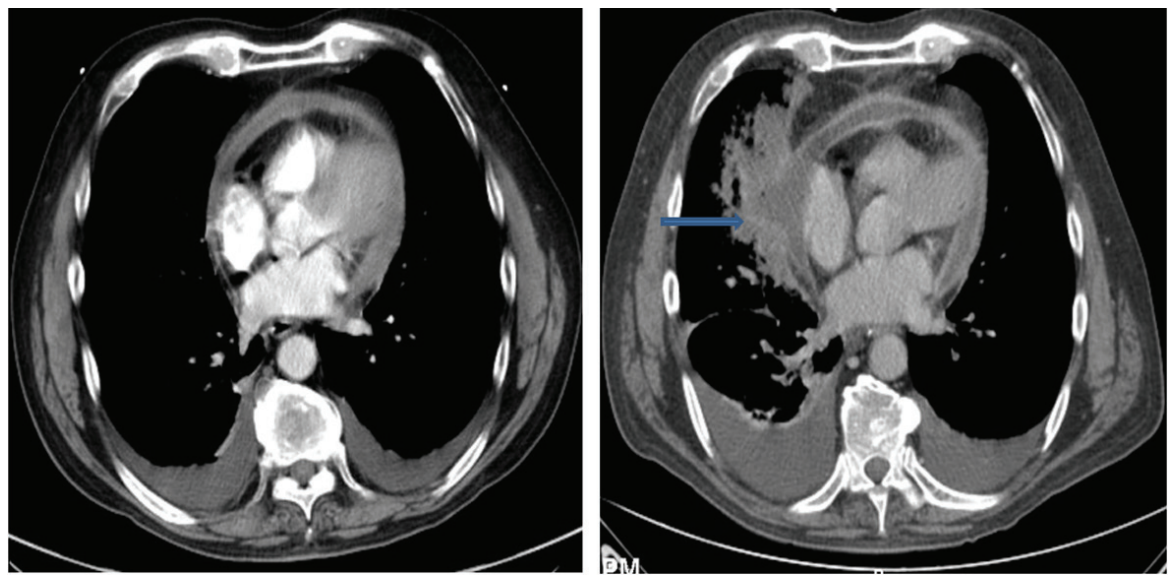

Figure 2. Computed tomography of chest showing right middle lobe consolidation, pericardial effusion and breach in pericardium abutting the right middle lobe (arrow). Notice the small pericardial effusion in the CT chest done 1 week ago (panel on left).

Initial laboratory studies demonstrated leukocytosis $(17$ $\mathrm{K} / \mu \mathrm{L}$ ), elevated ESR (46), and hypoalbuminemia (2.6 g/ dL). Renal function was normal. EKG showed sinus tachycardia with ST segment depression and T-wave inversion in all leads. Chest radiograph showed bilateral pleural effusion with airspace disease in the right middle and lower lobes which were new when compared with a radiograph obtained 2 days earlier (Fig. 1).

A trans-thoracic echocardiogram showed a small-tomoderate pericardial effusion circumferentially with no echocardiographic evidence of tamponade. Left ventricular systolic function was estimated to be normal. Computed tomographic (CT) scan of the chest showed right middle lobe consolidation with a right-sided pleural effusion. There was a pericardial effusion and pericardial enhancement consistent with pericarditis. There was also a breach of the pericardium abutting the right middle lobe of the lung (Fig. 2).

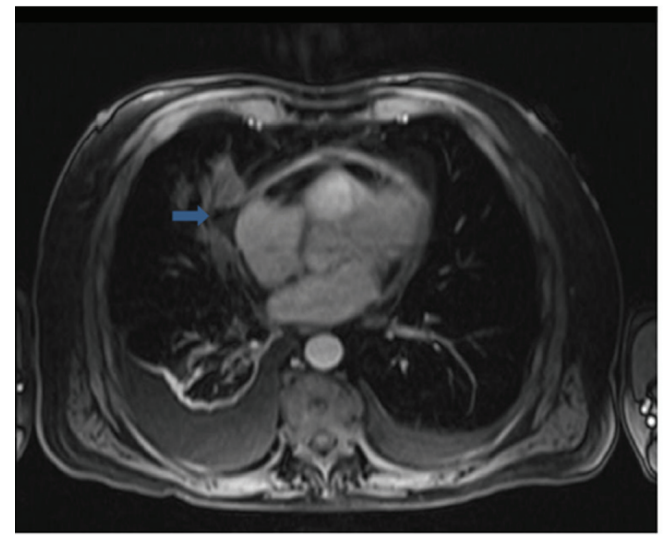

Figure 3. Cardiac MRI showing defect in pericardial continuity and focus of air within the pericardial sac adjacent to the area of lung consolidation (arrow).
Compared to a CT done 1 week earlier the pericardial effusion was larger and the pericardial defect and right middle lobe consolidation were both new. A cardiac MRI obtained 2 days later confirmed the $\mathrm{CT}$ findings and showed a significant decrease in the pericardial effusion compared to the $\mathrm{CT}$ chest and a focus of air within the pericardial sac adjacent to the area of consolidation, consistent with decompression of the pericardial fluid into the right pleural cavity and adjacent lung parenchyma (Fig. 3).

On analysis, the right pleural fluid was a neutrophilpredominant exudate. Both a tuberculin skin test and $\mathrm{TB}$ neutrophil gold test were negative. The ANA was positive at a titre of 1:160. The ds-DNA, anti-smith, RNP and antiCentromere antibodies and SCL-70 were negative. Pericardial biopsy and drainage were performed. Pericardial fluid cytology was negative for any malignant cells and culture was negative for any bacterial, fungal or tubercular elements. On pathologic examination of the pericardium there was an increase in non-specific fibrous connective tissue. Treatment for idiopathic pericarditis with NSAIDS initially and then colchicine was initiated. He improved over time, and a repeat echocardiogram showed resolution of the pericardial effusion.

\section{Discussion}

To our knowledge, this is the first reported case of spontaneous pericardial rupture into the lung and pleural space. It occurred in the absence of any history of trauma. From the sequence of events, we presume that the productive cough which immediately preceded this diagnosis represented the coughing up of pericardial fluid (pericardiohydroptysis). Aggressive work-up including pericardial biopsy failed to reveal a specific etiology. The patient recovered with conser- 
vative management.

Four major studies [2-5] have addressed the investigation of large pericardial effusions of unknown etiology. In the largest of these studies, $37 \%$ of patients with moderate and severe effusion had cardiac tamponade. The most common diagnosis was acute idiopathic pericarditis ( $20 \%$ of patients) followed by iatrogenic effusion (16\%), neoplastic effusion (13\%), and chronic idiopathic pericardial effusion (9\%) [3]. Our patient had acute inflammatory signs initially suggesting an acute inflammatory pericarditis. The non-specific biopsy and negative laboratory studies lead us to categorize this as an acute idiopathic pericarditis. Resolution with NSAIDS and Colchicine supports that diagnosis.

Rupture of the pericardium is a rare clinical finding traditionally considered to be indicative of an injury severe enough to crush or pierce the thoracic cage [6]. Most patients never reach the hospital, and those who do often have severe associated injuries [2]. A pericardial rupture should be suspected in a patient with severe blunt trauma to the chest [7]. However to our knowledge there are no prior reports of spontaneous non-traumatic pericardial rupture into the pleura and lung parenchyma [8]. The lack of a specific diagnosis such as tumor or infection which might cause local invasion and focal pericardial weakness makes the case even more unusual. In two series on large pericardial effusions tamponade was reported in $44 \%$ and $37 \%$ of patients respectively. There were no cases of pericardial rupture as a complication reported in them, the mechanism by which our patient's pericardium appears to have decompressed $[2,3]$.

The diagnosis of most pericardial ruptures described in literature was made intraoperatively as an incidental finding [9] or due to a high index of suspicion based on the nature of chest trauma. Once pericardial rupture is recognized, treatment is relatively simple and effective. Pericardial closure with nonabsorbable interrupted sutures is usually all that is needed [10]. A small opening should be left for drainage. A defect in the pericardium can be closed with a Dacron or polytetrafluoroethylene patch $[10,11]$. The prognosis of these patients depends on the management of their associated cardiac contusion and other injuries. If, however, the pericardial rupture is not recognized and treated it could belife threatening due to cardiac herniation $[12,13]$.

In our patient, the rent was small and closed spontaneously. Pericardial fluid was sampled, a biopsy taken, and a drain left in situ. Recovery was uneventful. A follow up echocardiogram done after 6 months showed complete resolution in the pericardial effusion.

\section{Conclusion}

To our knowledge, this is the only reported case of a pericardial rupture into the pleural space and lung parencyma from a non-traumatic cause. The progression or natural course of pericardial rupture is not known and hence the optimal man- agement not defined [14]. The case is unique as idiopathic pericarditis and pericardial effusion has been associated with pericardial tamponade as a common complication in most series and not pericardial rupture [15].

\section{References}

1. Mattila S, Silvola H, Ketonen P. Traumatic rupture of the pericardium with luxation of the heart. Case report and review of the literature. J Thorac Cardiovasc Surg. 1975;70(3):495-498.

2. Colombo A, Olson HG, Egan J, Gardin JM. Etiology and prognostic implications of a large pericardial effusion in men. Clin Cardiol. 1988;11(6):389-394.

3. Sagrista-Sauleda J, Merce J, Permanyer-Miralda G, Soler-Soler J. Clinical clues to the causes of large pericardial effusions. Am J Med. 2000;109(2):95-101.

4. Corey GR, Campbell PT, Van Trigt P, Kenney RT, O'Connor CM, Sheikh KH, Kisslo JA, et al. Etiology of large pericardial effusions. Am J Med. 1993;95(2):209213.

5. Levy PY, Fournier PE, Charrel R, Metras D, Habib G, Raoult D. Molecular analysis of pericardial fluid: a 7-year experience. Eur Heart J. 2006;27(16):1942-1946.

6. Clark DE, Wiles CS, 3rd, Lim MK, Dunham CM, Rodriguez A. Traumatic rupture of the pericardium. Surgery. 1983;93(4):495-503.

7. Fulda G, Brathwaite CE, Rodriguez A, Turney SZ, Dunham CM, Cowley RA. Blunt traumatic rupture of the heart and pericardium: a ten-year experience (19791989). J Trauma. 1991;31(2):167-172; discussion 172163.

8. Borrie J, Lichter I. Pericardial rupture from blunt chest trauma. Thorax. 1974;29(3):329-337.

9. Porte HL, Janecki-Delebecq TJ, Finzi L, Metois DG, Millaire A, Wurtz AJ. Pericardoscopy for primary management of pericardial effusion in cancer patients. Eur J Cardiothorac Surg. 1999;16(3):287-291.

10. Place RJ, Cavanaugh DG. Computed tomography to diagnose pericardial rupture. J Trauma. 1995;38(5):822823.

11. Permanyer-Miralda G, Sagrista-Sauleda J, SolerSoler J. Primary acute pericardial disease: a prospective series of 231 consecutive patients. Am J Cardiol. 1985;56(10):623-630.

12. Posner MR, Cohen GI, Skarin AT. Pericardial disease in patients with cancer. The differentiation of malignant from idiopathic and radiation-induced pericarditis. Am J Med. 1981;71(3):407-413.

13. Watkins BM, Buckley DC, Peschiera JL. Delayed presentation of pericardial rupture with luxation of the heart following blunt trauma: a case report. J Trauma. 1995;38(3):368-369. 
14. Soler-Soler J, Sagrista-Sauleda J, Permanyer-Miralda G. Relapsing pericarditis. Heart. 2004;90(11):1364-1368.

15. Restrepo CS, Lemos DF, Lemos JA, Velasquez E, Di- ethelm L, Ovella TA, Martinez S, et al. Imaging findings in cardiac tamponade with emphasis on CT. Radiographics. 2007;27(6):1595-1610. 